\title{
Progress of UNAIDS 90-90-90 targets in a district in KwaZulu-Natal, South Africa, with high HIV burden, in the HIPSS study: a household-based complex multilevel community survey

\author{
Anneke Grobler, Cherie Cawood, David Khanyile, Adrian Puren, Ayesha B M Kharsany
}

\section{Summary}

Background With the goal of eliminating new HIV infections, UNAIDS set the ambitious 90-90-90 targets to be achieved by 2020. We assessed whether these targets are being met among participants of the HIV Incidence Provincial Surveillance System (HIPSS) in a high-burden district of South Africa.

Methods We used data from a HIPSS household-based, cross-sectional survey of HIV prevalence and incidence done in the uMgungundlovu district, KwaZulu-Natal, in 2014 and 2015. In randomly selected enumeration areas, 50 households were drawn systematically along a serpentine pattern from a random start point. One eligible individual in each household was asked to provide blood for HIV testing and to complete a questionnaire. If a household refused to participate, the house next to it was approached. Eligible participants were aged 15-49 years, lived in the household, were not planning to move away, and spoke English or Zulu. Viral load was measured in samples positive for HIV. We also assessed participants HIV linkage to care and treatment. Data were population weighted to allow for multilevel sampling and non-response.

Findings 9812 participants were enrolled, 3547 men (36\%) and 6265 women (64\%). Overall, 504 of 1014 men (estimate 52\%, 95\% CI 47-56) and 1833 of 2955 women (65\%, 62-67) who were HIV positive knew their HIV status. Of those who knew, 344 of 522 men $(69 \%, 63-75)$ and 1254 of 1845 women $(70 \%, 68-73)$ were taking ART. Among recipients of ART, 294 of 341 men $(85 \%, 80-90)$ and 1117 of 1249 women $(90 \%, 87-92)$ had viral loads less than 1000 copies per mL. Among all HIV-positive participants, 427 of 1014 men (44\%, 39-49) and 1680 of 2955 women (58\%, 55-61) had viral loads lower than 1000 copies per $\mathrm{mL}$.

Interpretation No UNAIDS 90-90-90 targets had been met in our study population. Major campaigns are needed to increase HIV testing, especially among men, and to ensure all HIV-positive patients are taking ART.

Funding Centers for Disease Control and Prevention and the US Presidents Emergency Plan for AIDS Relief (PEPFAR).

\section{Introduction}

Antiretroviral therapy (ART) prevents HIV transmission, and in the HPTN052 study, ${ }^{2}$ provided $96 \%$ protection against transmission when the HIV-positive partner in a couple was treated. In another study of 767 HIVserodiscordant couples who did not use condoms, no HIV transmissions occurred when the HIV-positive person was taking ART and had achieved viral suppression. ${ }^{3}$ Undetectable HIV RNA reflects successful treatment, improved individual health, and correlates with reduced HIV transmission. ${ }^{4}$ Giving more HIVpositive people ART is expected to reduce HIV incidence at a population level. In an area of KwaZulu-Natal, South Africa, every $1 \%$ increase in coverage of ART has reduced HIV incidence by $1 \cdot 1 \%{ }^{5}$

Aiming to end the AIDS epidemic worldwide, UNAIDS has set ambitious targets of having $90 \%$ of people living with HIV knowing their HIV status, $90 \%$ of people diagnosed as having HIV receiving sustained ART, and $90 \%$ of people receiving ART having viral suppression by $2020 .{ }^{6}$ When these targets are met, $73 \%$ of all HIV-positive people would have viral suppression, which, according to modelling, would end the AIDS epidemic by 2030. Delivery of medical care to HIV-positive people requires a sequence of diagnostic tests, treatment with ART, and monitoring of viral load: the HIV linkage to care and treatment cascade. ${ }^{7.8}$

In South Africa, the HIV epidemic has substantial geographical variation, with KwaZulu-Natal having the highest burden and a provincial HIV prevalence in pregnant women of $40 \%$ (95\% CI 38-42). By contrast, in the Northern Cape, the province with the lowest burden, antenatal HIV prevalence was less than half of that in KwaZulu-Natal (18\%, 95\% CI 15-20). In addition to provincial variation, there is much heterogeneity at district level. Within KwaZulu-Natal, antenatal HIV prevalence varies from $46 \%$ (the highest nationally) in iLembe to $33 \%$ in Amajuba, although in all 12 districts in KwaZulu-Natal, HIV prevalence exceeds $30 \%$.

Despite 1.8 million people taking ART in South Africa, the high number of people needing treatment means that coverage is low (52\% in 2011). ${ }^{10}$ South Africa expanded
Lancet HIV 2017; 4: e505-13 Published Online August 2, 2017 http://dx.doi.org/10.1016 S2352-3018(17)30122-4

See Comment page e479 Centre for the AIDS Programme of Research in South Africa (CAPRISA), Durban, South Africa (A Grobler PhD, Prof A B M Kharsany PhD); Clinical Epidemiology and Biostatistics Unit, Murdoch Children's Research Institute, Melbourne, VIC, Australia (A Grobler); Epicentre AIDS Risk Management (Pty) Limited Paarl, South Africa (C Cawood MBA, D Khanyile BA); Centre for HIV and STIs, National Institute for Communicable Diseases, National Health Laboratory Service (NICD/NHLS), Johannesburg, South Africa (A Puren MBBCh); and Division of Virology, School of Pathology, University of Witwatersrand, Johannesburg, South Africa (A Puren)

Correspondence to: Dr Anneke Grobler, Centre for the AIDS Programme of Research in South Africa (CAPRISA), Doris Duke Medical Research Institute, Nelson R Mandela School of Medicine, University of KwaZulu-Natal, Private Bag X7,

Congella, 4013, Durban South Africa caprisa@caprisa.org 
Research in context

\section{Evidence before this study}

In 2014, UNAIDS proposed their 90-90-90 HIV testing and treatment targets for 2020. We searched PubMed with the search terms "90-90-90", "HIV", and "Africa" for all Englishlanguage papers reporting on these targets published from Jan 1, 2014, to May 31, 2016. Measuring the progress towards achieving these targets, however, is difficult. Most available data are not based on population-based random surveys and, therefore, do not provide unbiased population-level estimates. Rather, most information is derived from model-based estimates, self-reported HIV status, or data collected routinely in clinics.

\section{Added value of this study}

The first target, knowledge of HIV status, can only be measured in a population-based study and not with clinic-based data. The last target, viral suppression, cannot be based solely on patients attending ART clinics because it excludes patients who start
ART but in whom viral load has not been assessed. Our study overcame both these issues. We used data from a multilevel community survey to measure directly population-level knowledge of HIV status, use of ART, and viral suppression (based on self-reports and laboratory testing) to assess progress towards achieving the 90-90-90 targets in an area with a high HIV burden. The UNAIDS 90-90-90 targets were not met in our study population, mainly owing to lack of knowledge of HIV status, especially among men. The target best achieved was viral suppression by ART.

\section{Implications of all the available evidence} It is unlikely that the UNAIDS 90-90-90 targets will be met in most high-burden countries and areas by 2020 . Some isolated countries (eg, Botswana) or communities might reach these targets, but other countries, such as Kenya, are far from doing so. Major campaigns are needed to increase the prevalence of HIV testing, especially among men. access to ART by changing the eligibility criteria from a CD4 count less than 200 cells per $\mu \mathrm{L}$ to a count less than 350 cells per $\mu \mathrm{L}$ in $2013,{ }^{11}$ and again in 2015 , after this study had started, to less than 500 cells per $\mu \mathrm{L} .^{12}$ We assessed the HIV linkage to care and treatment cascade among participants enrolled in the HIV Incidence Provincial Surveillance System (HIPSS), a multilevel community-based survey, in KwaZulu-Natal, South Africa. We aimed to identify the points in the cascade where patients are lost to care, preventing them from achieving continuous HIV treatment and viral suppression.

\section{Methods}

\section{Study design and population}

The design of HIPSS has been described elsewhere. ${ }^{13}$ Briefly, a three-stage sampling design was used to randomly select a household-based representative sample of 10000 individuals in two cross-sectional surveys (appendix). In the first stage, 212 enumeration areas used for the national census were drawn randomly. In the second stage, from each enumeration area 50 households were drawn systematically along a serpentine pattern from a random start point. These households were approached, and if a household refused, the house next to it was approached instead. In the third stage, one member of each selected household was selected at random. For this analysis, we used data from a HIPSS cross-sectional survey done from June 11, 2014, to June 22, 2015, in Vulindlela and Greater Edendale in uMgungundlovu District, central KwaZulu-Natal, where the HIV prevalence among pregnant women was $42 \%$ (95\% CI 35-51)-one of six districts in South Africa with antenatal HIV prevalence greater than $40 \% .{ }^{9}$ The estimated population in the study area was 117976 men and 126723 women; $48 \%$ of the population was male.
Eligible participants were men and women aged 15-49 years residing in the selected households who were willing to participate and undergo study procedures, and could provide written informed consent in either English or Zulu (or parental consent with assent if participants were $<18$ years). We excluded individuals who did not have the mental capacity to provide consent or who were planning to relocate within 12 months.

Selected individuals were interviewed by fieldworkers with use of a standardised questionnaire with closedended questions about demographic and socioeconomic characteristics, behavioural and medical information, HIV status, sexual risk behaviour, and access to health care. Women were also asked about childbearing history and access to prevention of mother-to-child transmission services, and men were additionally asked about circumcision status.

Participants provided plasma, urine, and genital swab samples to allow testing for HIV, sexually transmitted infections, and pregnancy. HIV testing was done with fourth generation EIAs, ${ }^{14}$ with HIV antibodies and antigens measured by the Biomérieux Vironostika Uniform II Antigen/Antibody Microelisa system (BioMérieux, Marcy I'Etoile, France) and HIV 1/2 Combi Roche Elecsys (Roche Diagnostics, Penzberg, Germany). Positive samples were confirmed with an HIV-1 western blot assay (Bio-Rad Laboratories, Redmond, WA, USA). Individual HIV-1 RNA viral load measurements were tested with Roche COBAS Ampliprep/ COBAS TaqMan HIV-1 version 2.0 assay (TaqMan, Roche Diagnostics, Mannheim, Germany). In HIV-positive participants, CD4 cell counts were done with flow cytometry (BD FACSCalibur, BD Biosciences, San Jose, CA, USA) and viral load was measured with the COBAS AmpliPrep/COBAS TaqMan HIV-1 version 2.0 
assay. Testing for presence of ART (nevirapine, efavirenz, lamivudine, emtricitabine, zidovudine, lopinavir, tenofovir) was done by high-performance liquid chromatography coupled to tandem mass spectrometry in HIV-positive participants who had high viral loads ( $>10000$ copies per $\mathrm{mL}$ ) but who reported taking ART or in individuals who had undetectable viral loads but reported not taking ART.

Ethics approval for HIPSS was provided by the University of KwaZulu-Natal biomedical research ethics committee (BF269/13) on May 13, 2014, and the US Centers for Disease Control and Prevention in collaboration with the Department of Health, Province of KwaZulu-Natal (HRKM 08/14). Participants gave written informed consent before enrolment.

\section{Statistical analysis}

The sample for this study consisted of all participants enrolled in the HIPSS survey who provided peripheral blood samples for HIV testing and completed interview questions. The elements of the UNAIDS 90-90-90 treatment cascade were assessed in the following ways: the numerator for HIV status was the number of participants who had positive HIV tests and self-reported in the survey that they were HIV positive during the interview, and the denominator was the number of participants who tested HIV positive in the survey; for receipt of sustained ART, the numerator was the number of participants who self-reported using ART in the interview and the denominator was the number of participants with laboratory confirmed HIV and who reported knowing they were HIV positive in the interview; for viral suppression, the numerator was the number of participants who self-reported using ART in the interview and had laboratory confirmed HIV RNA viral loads less than 1000 copies per $\mathrm{mL}$, and the denominator was the number of participants who self-reported using ART. For receipt of sustained ART, we also did an analysis in which participants with laboratory confirmed presence of ART were included in the numerator. All participants who reported using ART were assumed to be retained in the cascade of care. Participants were classified as being linked to care if they reported receiving at least one of home-based care, a CD4 cell count, viral-load testing, and ART or treatment of opportunistic infections. We defined HIV-positive participants as those who tested positive in samples provided for this study.

To account for the complex multilevel sampling design, weighted proportions and SEs were analysed with SAS

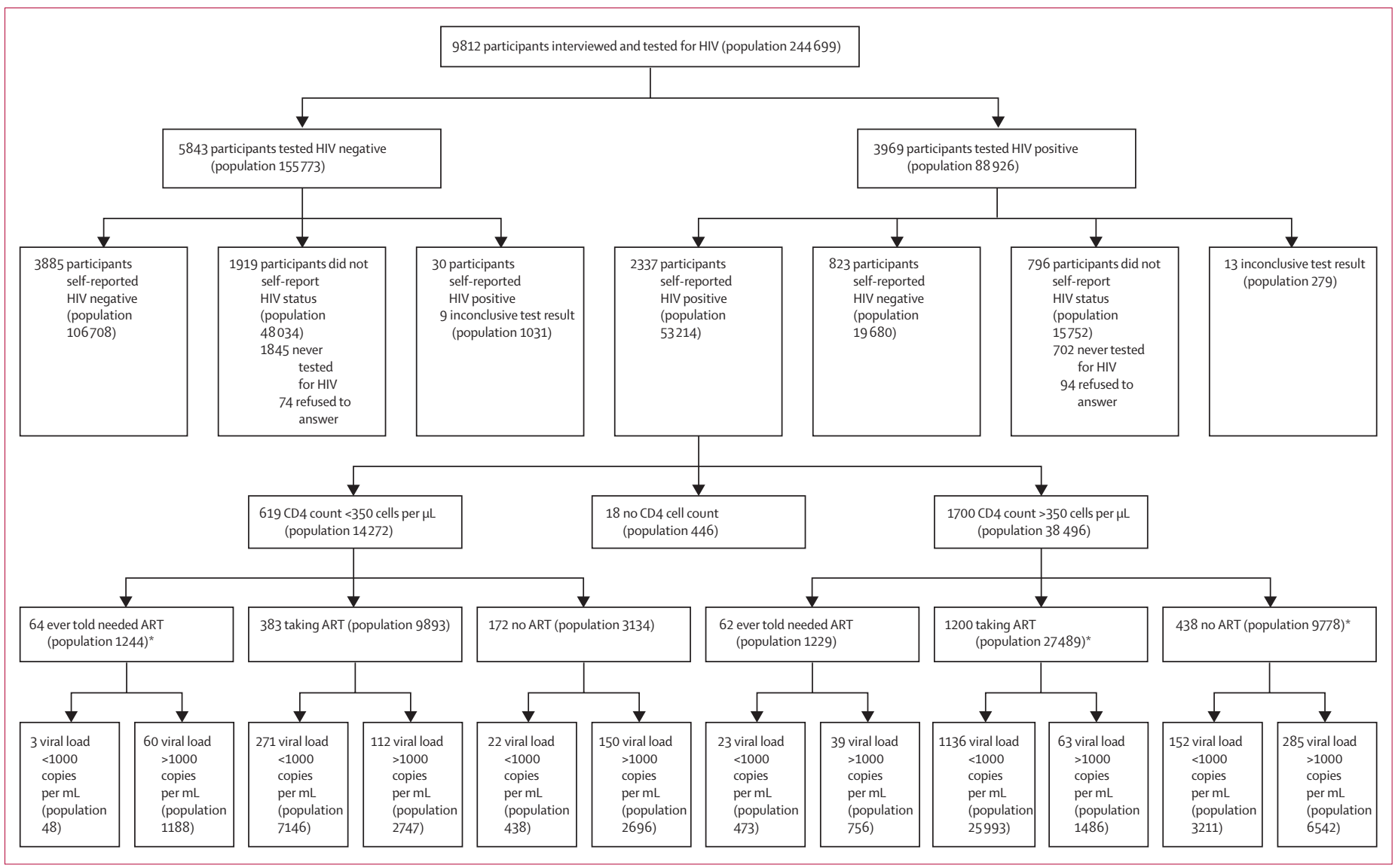

Figure 1: HIV status by laboratory testing, knowledge of HIV status, CD4 cell counts, ART status, and viral load suppression status

Calculations were weighted; due to rounding the sum of some population numbers do not equal the total on the previous level. ART=antiretroviral therapy. ${ }^{*}$ One viral load missing. 
(version 9.4) survey procedures (appendix). Weights took into account the probability of selecting the enumeration area, the household in the enumeration area, and the individual in the household, then were adjusted for nonresponse and benchmarked to the size of the population in the study area. For comparisons of weighted proportions, we calculated $\mathrm{p}$ values with the Rao-Scott $\chi^{2}$ statistic, a design-adjusted version of the Pearson $\chi^{2}$ statistic, using a

\begin{tabular}{|c|c|c|}
\hline & $\begin{array}{l}\text { Women } \\
(n=6265)\end{array}$ & $\begin{array}{l}\text { Men } \\
(n=3547)\end{array}$ \\
\hline \multicolumn{3}{|l|}{ Marital status } \\
\hline Married or living together & $857(14 \%)$ & $241(8 \%)$ \\
\hline Single & $5408(86 \%)$ & $3306(92 \%)$ \\
\hline \multicolumn{3}{|l|}{ Age group (years) } \\
\hline $15-19$ & $958(18 \%)$ & $658(20 \%)$ \\
\hline $20-24$ & $1266(20 \%)$ & $814(21 \%)$ \\
\hline $25-29$ & $1087(18 \%)$ & $602(18 \%)$ \\
\hline $30-34$ & $833(14 \%)$ & $461(14 \%)$ \\
\hline $35-39$ & $760(12 \%)$ & $405(12 \%)$ \\
\hline $40-44$ & $660(10 \%)$ & $320(9 \%)$ \\
\hline $45-49$ & $701(9 \%)$ & $287(7 \%)$ \\
\hline \multicolumn{3}{|l|}{ Education } \\
\hline No schooling or before primary & $265(3 \%)$ & $153(3 \%)$ \\
\hline Primary (grade $1-7$ ) & $375(6 \%)$ & $232(7 \%)$ \\
\hline $\begin{array}{l}\text { Incomplete secondary (grade 8-11 } \\
\text { or NQF level 3) }\end{array}$ & $2674(45 \%)$ & $1547(47 \%)$ \\
\hline $\begin{array}{l}\text { Completed secondary (grade } 12 \text { or } \\
\text { NQF 4) }\end{array}$ & $2603(41 \%)$ & $1406(38 \%)$ \\
\hline Tertiary (diploma/degree) & $345(5 \%)$ & $207(5 \%)$ \\
\hline No response & $3(<0 \cdot 5 \%)$ & $2(<0 \cdot 6 \%)$ \\
\hline African origin & $6257(>99 \%)$ & 3545 (>99\%) \\
\hline Ever diagnosed with an STI & $318(6 \%)$ & $231(8 \%)$ \\
\hline Ever had sex & $5447(86 \%)$ & $2855(81 \%)$ \\
\hline $\begin{array}{l}\text { Away from home for }>1 \text { month in } \\
\text { the past } 12 \text { months }\end{array}$ & $623(10 \%)$ & $398(11 \%)$ \\
\hline Ever been pregnant & $4391(71 \%)$ & NA \\
\hline \multicolumn{3}{|c|}{$\begin{array}{l}\text { NQF=National Qualifications Framework. STI=sexually transmitted infection. } \\
N A=\text { not applicable. }\end{array}$} \\
\hline
\end{tabular}

design correction based on the design effects of the proportions, which involved differences between observed and expected frequencies. ${ }^{15,16}$ Estimates used a weighted likelihood function, weighted first derivatives, and the Newton-Raphson algorithm to find the coefficient estimates that maximised the weighted pseudolikelihood. We used the Taylor series method to estimate SEs of proportions and design-based CIs and test statistics. Descriptive statistics, such as population-weighted percentages, were weighted and are cited with 95\% CIs. Unweighted counts are also presented. ${ }^{17}$ Multivariate logistic regression was done to investigate factors associated with meeting each of the steps of the cascade.

\section{Role of the funding source}

The funders of the study played a role in the study design, data interpretation and writing of the report, but not in data collection or data analysis. The corresponding author had full access to all the data in the study and had final responsibility for the decision to submit for publication.

\section{Results}

Of 14618 households approached for the HIPSS survey, 11289 agreed to participate. 10389 households (92\%) had eligible participants, 577 (6\%) of whom refused to participate. Thus, 9812 participants were enrolled in HIPSS and tested for HIV (figure 1), of whom 3547 (36\%) were men and 6265 (64\%) were women (table 1).

3969 of 9812 people overall were confirmed to be HIV positive (36\%, 95\% CI 35-38). The population-weighted HIV prevalence for men was $28 \%$ (95\% CI 26-30, 1014 of 3547) and for women was 44\% (42-46, 2955 of 6265 , $\mathrm{p}<0 \cdot 0001)$. Thus, we estimate that 33045 HIV-positive men and 55881 HIV-positive women aged 15-49 years were living in the overall study area. 31\% of men (95\% CI $29-33,1221$ of 3547$)$ and $18 \%$ of women $(16-20,1326$ of 6265) self-reported never having been tested for HIV. $52 \%$ of men (49-54, 1719 of 3547$)$ and $52 \%$ of women (50-54, 2989 of 6265) reported being HIV negative. The remainder of participants (85 women and 105 men) refused to disclose HIV status to data collectors.

\begin{tabular}{|c|c|c|c|c|c|c|c|c|c|}
\hline & \multicolumn{3}{|l|}{ Men } & \multicolumn{3}{|l|}{ Women } & \multicolumn{3}{|l|}{ All } \\
\hline & Number & Population & $\begin{array}{l}\text { Proportion } \\
(95 \% \mathrm{Cl})\end{array}$ & Number & Population & $\begin{array}{l}\text { Proportion } \\
(95 \% \mathrm{Cl})\end{array}$ & Number & Population & $\begin{array}{l}\text { Proportion } \\
(95 \% \mathrm{Cl})\end{array}$ \\
\hline Received nutritional support & 173 & 6440 & $37 \%(29-44)$ & 598 & 13247 & $36 \%(31-41)$ & 771 & 19687 & $36 \%(31-41)$ \\
\hline Had a treatment buddy & 123 & 4116 & $23 \%(16-31)$ & 422 & 7764 & $21 \%(18-25)$ & 545 & 11880 & $22 \%(18-26)$ \\
\hline Home-based care & 47 & 2109 & $12 \%(6-18)$ & 182 & 3504 & $10 \%(7-12)$ & 229 & 5613 & $10 \%(8-13)$ \\
\hline Had a CD4 cell count & 453 & 15918 & $90 \%(87-93)$ & 1679 & 33830 & $93 \%(91-94)$ & 2132 & 49748 & $92 \%(90-94)$ \\
\hline Received a viral-load test & 380 & 13402 & $76 \%(71-81)$ & 1409 & 28171 & $77 \%(74-81)$ & 1789 & 41573 & $77 \%(74-80)$ \\
\hline Treatment of opportunistic infections & 155 & 5326 & $30 \%(25-36)$ & 485 & 11007 & $30 \%(27-34)$ & 640 & 16333 & $30 \%(27-34)$ \\
\hline Linked to care* & 488 & 16806 & $95 \%(93-97)$ & 1762 & 35065 & $96 \%(95-97)$ & 2250 & 51870 & $96 \%(95-97)$ \\
\hline
\end{tabular}


2367 of 9748 participants (22\%, 95\% CI 21-24) selfreported that they were HIV positive, making the population-weighted prevalence $15 \%$ for men (13-17, 522 of 3529 ) and $29 \%$ for women (27-31, 1845 of 6219; $\mathrm{p}<0 \cdot 0001)$. The proportions of participants accurately self-reporting their status as HIV positive in the interviews were $52 \%$ of men (47-56, 504 of 1014) and $65 \%$ of women (62-67, 1833 of 2955; p<0.0001). Among the 1014 HIVpositive men, $260(23 \%, 19-26)$ self-reported to have never been tested and 205 (22\%, 19-25) self-reported to have tested HIV negative in their latest HIV test (median time since latest test before survey 19 months, IQR 9-39). Among the 2955 HIV-positive women, 442 (11\%, 9-13) self-reported to have never been tested and 618 (22\%, 20-25) self-reported to have tested HIV negative in their latest HIV test (median time since testing 13 months, IQR 5-27). The remainder of participants with confirmed HIV-positive status (45 men and 62 women) refused to disclose HIV status to data collectors.

Of 4930 women who had ever been tested for HIV, 3918 (79\%, 95\% CI 78-81) had ever been pregnant, and among 1326 who had never been tested, 473 (32\%, 28-36) had ever been pregnant $(\mathrm{p}<0 \cdot 0001) .38 \%$ of HIV positive women (35-41, 672 of 1845) also reported receiving an HIV test at an antenatal visit. If these women are removed from the analysis, 20\% of women (18-23, 1326 of 5593) had never been tested for HIV. Among women who had ever or never been pregnant, $8 \%(7-10,3473$ of $4391)$ and $42 \%$ (38-46, 853 of 1865), respectively, had never been tested for HIV.

Among participants who knew their HIV-positive status, almost all were linked to care (table 2, figure 2, appendix). The services accessed by most participants were CD4 cell counts and viral load testing (table 2).

Only $69 \%$ of men (95\% CI 63-75, 344 of 522) and $70 \%$ of women $(68-73,1254$ of 1845$)$ who knew they were HIV positive were taking ART $(\mathrm{p}=0.71)$ and, respectively, among these only 5\% (3-7, 32 of 522) and 5\% (4-6, 97 of 1845 ) reported being told by a nurse or doctor that they needed ART but were not currently receiving treatment. Of all HIV-positive participants, irrespective of CD4 cell count or knowledge of HIV status, 1592 of 3969 (42\%, 95\% CI 40-45) reported taking ART. Among those with confirmed HIV-positive status, 341 of 1014 men (37\%, 32-41) and 1251 of 2955 women (46\%, 43-48) were taking ART $(\mathrm{p}=0 \cdot 0004)$. When laboratory results for the presence of ART were included, the percentage of HIVpositive participants receiving ART remained similar (36\% of men, 31-40, 331 of 1014, and $46 \%$ of women, 43-48, 1251 of 2955; $\mathrm{p}<0 \cdot 0001)$.

Considering CD4-cell-count thresholds for starting ART, $74 \%$ of men (95\% CI 66-82, 144 of 215 ) and $65 \%$ of women (59-71, 239 of 404) who had CD4 counts less than 350 cells per $\mu \mathrm{L}$ reported taking ART. Similarly, $75 \%$ of men (69-81, 226 of 330) and 66\% of women (62-71, 506 of 802) who had CD4 counts less than 500 cells per $\mu \mathrm{L}$ reported taking ART.
Among HIV-positive participants, 2107 of 3969 (53\%, 95\% CI 50-56) had viral loads less than 1000 copies per $\mathrm{mL}$, irrespective of ART status, with prevalence being $44 \%$ (39-49, 427 of 1014) among men and 58\% (55-61, 1680 of 2955) among women $(\mathrm{p}<0 \cdot 0001)$. Among participants who reported taking ART, the proportion with viral loads less than 1000 copies per mL was not significantly different between men and women $(85 \%$, 80-90, 294 of 341 vs 90\%, 87-92, 1117 of 1249; $\mathrm{p}=0 \cdot 12$ ). When laboratory results for the presence of ART were included, the percentages become $89 \%$ (85-94, 298 of $331)$ and $92 \%(90-94,1156$ of 1248$)$, respectively $(\mathrm{p}=0 \cdot 28)$. $20 \%$ of men (16-24, 133 of 669$)$ and $32 \%$ of women (29-36, 563 of 1697) who were HIV positive and did not report taking ART had suppressed viral loads $(\mathrm{p}<0 \cdot 0001)$.

For the first target of the UNAIDS 90-90-90 cascade, knowledge of HIV-positive status, attainment in men and women was much lower among people younger than 25 years than among those in older age groups (figure 3, appendix), and for the second element, people who are HIV positive taking ART, attainment was lowest for participants aged 20-24 years. The third element of suppressed viral load was achieved in high proportions of all age groups.

Factors significantly associated with attainment of the overall UNAIDS 90-90-90 targets included female sex, being married or cohabitating, and age 35 years or older (table 3). The same variables were also significantly associated with improved attainment of the first target, which is unsurprising because this target largely affects overall target achievement.

\section{Discussion}

None of the UNAIDS 90-90-90 targets for diagnosis, treatment, and viral suppression were met among HIV-positive participants in our study population. In women, one target was met, with $90 \%$ taking ART

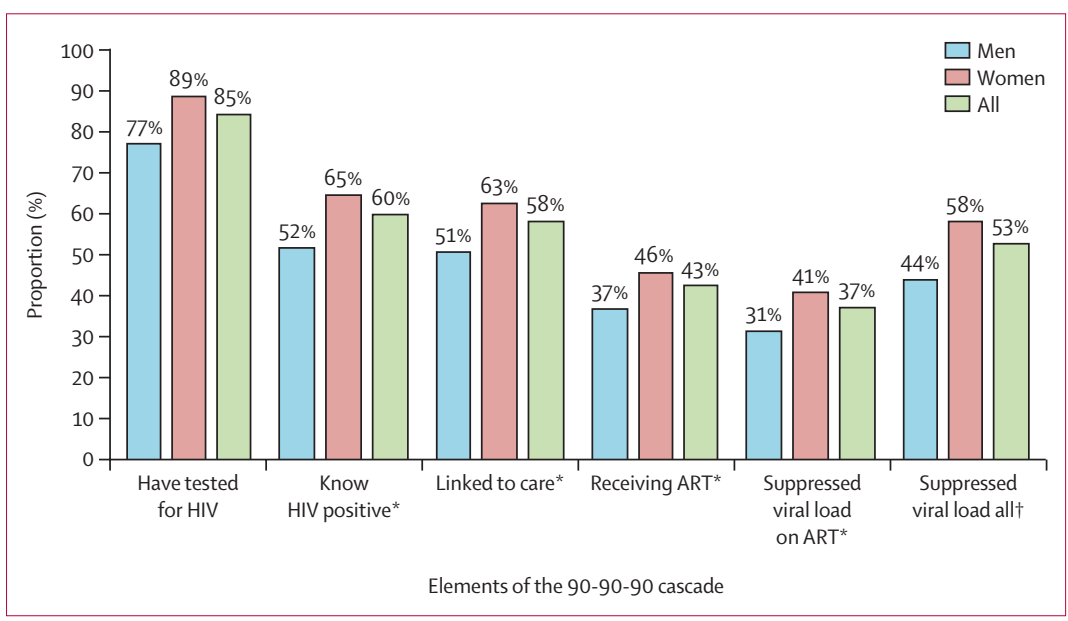

Figure 2: Cascade of care in participants

Percentages are based on the number of HIV-positive people and are population weighted. Absolute numbers for each element are available in the appendix. ART=antiretroviral therapy. *Subset of the element or elements to the left. †Denominator includes HIV-positive people with suppressed viral loads, irrespective of ART status. 
having suppressed viral load. This target had also been achieved in $85 \%$ of men. By contrast, only $52 \%$ of HIVpositive men and $65 \%$ of HIV-positive women knew their status, and, of those, $69 \%$ and $70 \%$, respectively, reported receiving ART. Only $44 \%$ of HIV-positive men and $58 \%$ of HIV-positive women had viral loads less than 1000 copies per $\mathrm{mL}$. The largest shortfall in the linkage to care and treatment cascade, therefore, was the first target, which is the entry point to the health-care system.

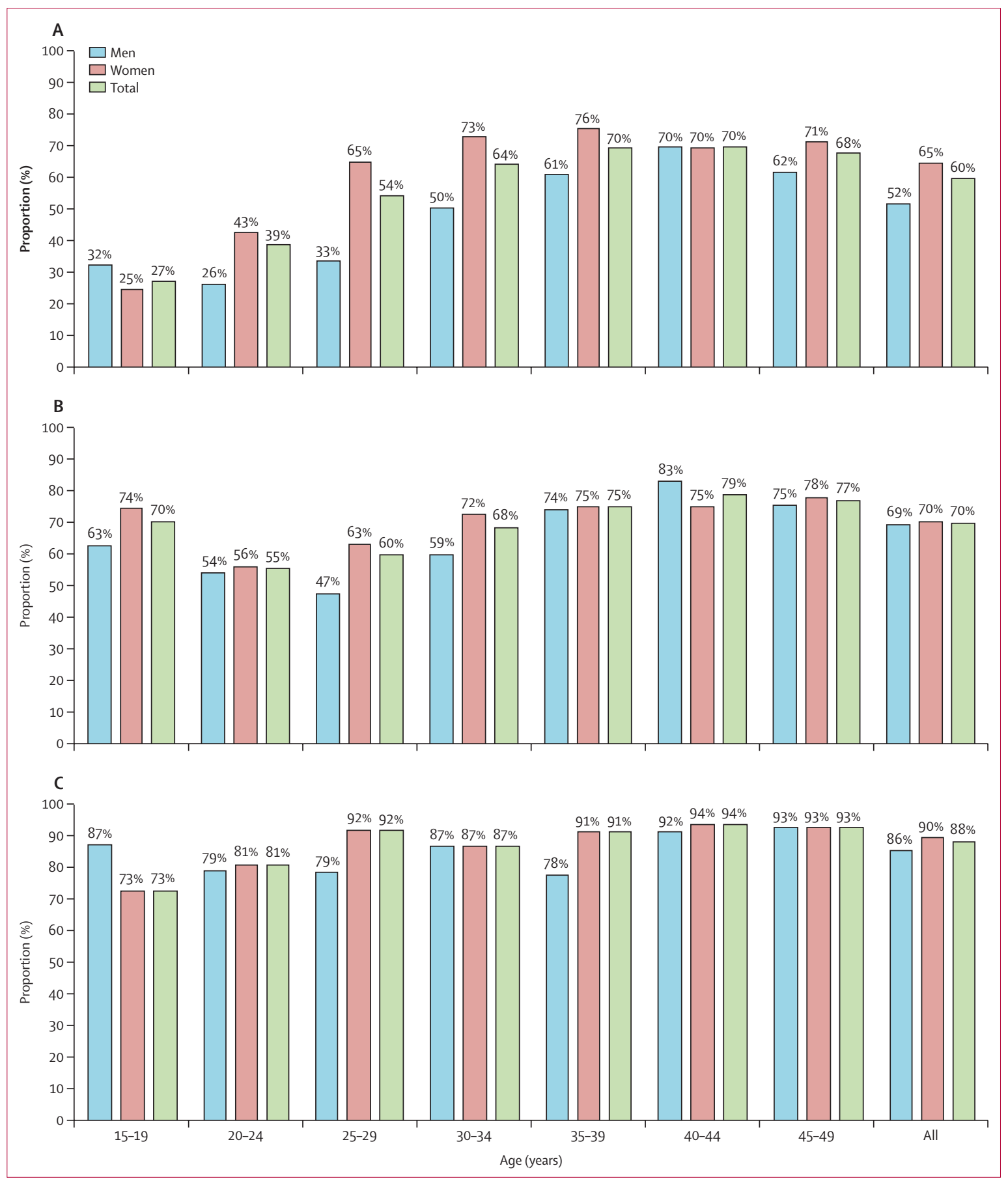

Figure 3: HIV cascade by age group

(A) People living with HIV who know they are HIV positive. (B) People who know they are HIV positive and are taking ART. (C) HIV-positive people taking ART with viral suppression. The absolute numbers for each age group are available in the appendix. ART=antiretroviral therapy. 


\begin{tabular}{|c|c|c|c|c|}
\hline & $\begin{array}{l}\text { People with HIV who } \\
\text { know their status }\end{array}$ & Taking ART* & Suppressed viral load $\dagger$ & $\begin{array}{l}\text { Overall suppressed } \\
\text { viral load } \ddagger\end{array}$ \\
\hline Sex (women vs men) & $2 \cdot 12(1 \cdot 75-2 \cdot 57)$ & $1.17(0.86-1.58)$ & $1.60(0.92-2 \cdot 77)$ & $2 \cdot 11(1.69-2.63)$ \\
\hline Marital status (married or living together vs single) & $1.51(1.12-2.04)$ & $1.09(0.81-1.48)$ & $1.13(0.56-2 \cdot 27)$ & $1.50(1.08-2.09)$ \\
\hline \multicolumn{5}{|l|}{ Age (years) } \\
\hline $15-19$ vs $45-49$ & $0.16(0.09-0.29)$ & $0.82(0.27-2.51)$ & $0.27(0.07-1.13)$ & $0.22(0.13-0.38)$ \\
\hline $20-24$ vs $45-49$ & $0.31(0.20-0 \cdot 47)$ & $0.37(0.23-0.61)$ & $0.28(0.12-0.68)$ & $0.23(0.16-0.34)$ \\
\hline $25-29$ vs $45-49$ & $0.62(0.41-0.93)$ & $0.44(0.27-0.72)$ & $0.60(0.23-1.58)$ & $0.32(0.24-0.44)$ \\
\hline $30-34$ vs $45-49$ & $0.94(0.67-1 \cdot 33)$ & $0.68(0.44-1.03)$ & $0.47(0.21-1 \cdot 05)$ & $0.51(0.37-0.70)$ \\
\hline $35-39$ vs $45-49$ & $1.22(0.85-1 \cdot 75)$ & $0.91(0.57-1.44)$ & $0.48(0.21-1.09)$ & $0.76(0.53-1.08)$ \\
\hline $40-44$ vs $45-49$ & $1.21(0.82-1 \cdot 78)$ & $1.10(0.67-1 \cdot 83)$ & $1.00(0 \cdot 45-2 \cdot 23)$ & $0.94(0.65-1.36)$ \\
\hline \multicolumn{5}{|l|}{ Education } \\
\hline Completed secondary & $0.84(0.57-1.22)$ & $0.82(0.48-1 \cdot 39)$ & $1 \cdot 35(0 \cdot 67-2 \cdot 72)$ & $1.05(0 \cdot 74-1 \cdot 50)$ \\
\hline Incomplete secondary & $1.44(0.97-2.15)$ & $0.82(0.50-1.34)$ & $1.03(0.55-1.94)$ & $1.19(0.80-1.75)$ \\
\hline No schooling & $0.58(0.32-1.05)$ & $0.65(0.30-1.41)$ & $6 \cdot 26(1 \cdot 39-28 \cdot 27)$ & $1.06(0.61-1.85)$ \\
\hline Primary schooling (ref) & 1 & 1 & 1 & 1 \\
\hline $\begin{array}{l}\text { Away from home for }>1 \text { month in past } 12 \text { months } \\
\text { (no vs yes) }\end{array}$ & $1.00(0.74-1 \cdot 36)$ & $0.95(0.66-1.38)$ & $1 \cdot 81(1.13-2 \cdot 91)$ & $1.29(0.96-1.73)$ \\
\hline \multicolumn{5}{|c|}{$\begin{array}{l}\text { ART=antiretroviral therapy. *Among HIV-positive people who know their status. †Among HIV-positive people taking ART. \#lrrespective of ART status or knowledge of HIV } \\
\text { status (among all HIV-positive people). }\end{array}$} \\
\hline
\end{tabular}

UNAIDS has estimated that only $45 \%$ of people living with HIV in sub-Saharan Africa know their status. ${ }^{6}$ In our study $60 \%$ of HIV-positive participants knew their status. Among HIV-infected populations in other countries, $83 \%$ in Botswana in $2013-15,{ }^{18} 77 \%$ in Malawi in $2013,{ }^{19} 59 \%$ in Kenya in $2012,{ }^{20}$ and $67 \%$ in Swaziland ${ }^{21}$ have been reported to know their HIV status. A small study in Cape Town, South Africa, in 2010 showed that $55 \%$ of HIV-positive people knew their HIV status. ${ }^{22}$ When separated by sex, $52 \%$ of HIV-positive men and $65 \%$ of HIV-positive women in our study reported knowing they were HIV positive. Shisana and colleagues ${ }^{23}$ found even lower proportions in South Africa in 2012 (38\% of HIV-positive men and 55\% of HIVpositive women). In Zambia in 2011-12, 52\% of men reported ever having been tested for HIV. ${ }^{24}$ Older studies have generally reported poorer knowledge of HIV status, which suggests that availability of HIV testing has increased over time. Botswana is the sub-Saharan country with the highest frequency of HIV diagnosis, reasons for which include high rates of testing in pregnant women and a policy of routine opt-out testing in health-care settings since 2004. ${ }^{18}$

Once people in the KwaZulu-Natal district in our study knew they were HIV positive, linking to the care and treatment cascade was good, with more than $90 \%$ having had CD4 cell counts and more than 95\% having ever linked to care. The results for the second UNAIDS 90-90-90 target, therefore, were $69 \%$ of men and $70 \%$ of women receiving ART. By contrast, in Swaziland, 50\% of HIV-positive participants were linked to care. ${ }^{21}$

$42 \%$ of all HIV-positive people in this study (37\% of men and $46 \%$ of women) reported taking ART, which is higher than the UNAIDS estimate of $39 \%$ of people living with HIV in sub-Saharan Africa. ${ }^{6}$ Additionally, Shisana and colleagues ${ }^{23}$ reported that $31 \%$ of HIV-positive people in South Africa $26 \%$ men and $35 \%$ women) were taking ART, and Takuva and colleagues $^{25}$ estimated that $34 \%$ were receiving ART. The PopART study ${ }^{26}$ found that $52 \%$ and $49 \%$ of HIV-positive people were taking ART in an area of the Western Cape province of South Africa and in Zambia, respectively. Other studies found that $63 \%$ of HIV-positive people in Malawi ${ }^{19}$ and $40 \%$ in Kenya ${ }^{20}$ were taking ART. Variability in coverage is seen across the entire continent with, for example, coverage of $41 \%$ in eastern and southern Africa, $23 \%$ in western and central Africa, and $19 \%$ in north Africa. ${ }^{6}$ One reason for the low ART coverage in most sub-Saharan African countries is the decision to give patients ART based on CD4 cell counts, meaning that many people diagnosed as having HIV were linked to care but ART was not started. Most countries are implementing test-and-treat systems, meaning that ART is started irrespective of CD4 cell count. The number of HIV-positive individuals taking ART is, therefore, expected to increase.

Viral load suppression was seen in $44 \%$ and $58 \%$ of HIV-infected men and women, respectively, in this study, which is substantially below the UNAIDS target of $73 \%$. In sub-Saharan Africa, the proportion of people living with HIV with suppressed viral loads is estimated to be 29-32\%. ${ }^{6,27}$ In Swaziland in 2011 the proportion was 35\%, in Botswana in 2013-15 it was 70\%, ${ }^{18}$ in Kenya in 2012 it was $40 \%,{ }^{20}$ and in South Africa in 2012 it was 25\%. ${ }^{25}$ Thus, despite high prevalence in the district in South Africa that we assessed, viral suppression was achieved 
in more people than in most other countries in the region. This finding can be partly attributed to our survey being done more recently than the other studies, because HIV diagnosis and ART coverage have improved over time. Additionally, many HIV-positive participants claimed not to be taking ART but had viral load suppression.

The greatest success in KwaZulu-Natal was the high proportions of participants taking ART who had achieved viral suppression, with $85 \%$ of men and $90 \%$ of women having viral loads less than 1000 copies per mL. These proportions are higher than in many developed countries, ${ }^{27}$ although lower than the $97 \%$ reported in Botswana. ${ }^{18}$ It was also much higher than the $26 \%$ reported in rural areas of the North West province of South Africa. ${ }^{28}$ We assessed ART use by asking participants whether they were currently taking ART. In the analysis of viral suppression, we did not include patients who had started but discontinued ART. We did not collect information on adherence to ART, but the high frequency of viral suppression suggests that it is good. ART is available free of charge at clinics close to where many people in the study area live. The public health system mandates monitoring of viral load to assess treatment success and detect issues with adherence, along with steps to be taken when patients stop taking treatment. ${ }^{29}$ These steps include comprehensive adherence counselling, repeated viral-load testing, and switching to second-line treatment. ${ }^{12}$

None of the three UNAIDS 90-90-90 cascade targets had been met in the population we assessed. If service coverage is not increased, South Africa will not be able to reverse the HIV epidemic in this high-burden district. However, the proportions of people who know their HIV status, are receiving ART, and have achieved viral load suppression are all greater than those in a representative sample of South Africa, ${ }^{23}$ which suggests that other districts face similar problems.

Knowledge of HIV status was the only target for which the proportion was higher in women than in men. This difference is driven by the testing of women during pregnancy. Men unaware of their HIV status are not linking to care, although once in care they seem to achieve the targets as well as women.

This study was based on a representative sample of the general population in the uMgungundlovu district and, therefore, our findings are generalisable to the whole study area. The greatest limitation of our study is that knowledge of HIV status and ART use were self-reported and, therefore, the data might be susceptible to bias, such as social desirability bias. For example, among 79 participants who reported being HIV negative but had positive HIV tests and were selected for laboratory ART testing, 31 tested positive for the presence of ART. If knowledge of HIV status was under-reported, the proportions of participants who knew their HIV status is an underestimate. Adherence to ART was not assessed and, therefore, we do not know whether participants who said they were taking ART were receiving adequate doses to achieve viral suppression. Finally, we only assessed people aged 15-49 years, meaning that the results are not generalisable to older or younger people.

Improvement is needed in all aspects of the HIV care and treatment cascade, with emphasis on campaigns to increase HIV testing. Such campaigns should be strengthened by scaling-up of testing in all settings, especially where men and boys can be reached such as mobile testing in communities ${ }^{30}$ and workplace testing. The PopART study ${ }^{31}$ showed increased uptake of testing was when it was offered in peoples' homes. For instance, one round of home-based testing led to an increase in people knowing their HIV status from $47 \%$ to more than $87 \%$.Because HIV testing is done before male circumcision, combining HIV and circumcision campaigns would be cost effective. In our study, many people who claimed to have been tested for HIV reported being HIV negative, yet they had HIV-positive results when tested during the study. This finding indicates the need for repeated testing.

\section{Contributors}

AK is the principal investigator of the study. AK, CC, and DK were responsible for the field work and quality assurance, AG for statistical analyses, and AP for laboratory testing and quality assurance. AG wrote the first draft of the manuscript. All authors reviewed and contributed to the final version of the manuscript.

\section{Declaration of interests}

We declare no competing interests.

\section{Acknowledgments}

The HIV Incidence Provincial Surveillance System was funded by the Centers for Disease Control and Prevention under terms of the cooperative agreement 3U2GGH000372-02W1 and the US Presidents Emergency Plan for AIDS Relief (PEPFAR). We thank May Zuma-Makhonza and Sithembile Dlamini, uMgungundlovu Health District, members of the HIV and AIDS/STI/TB (HAST) unit, Leslie Sakuneka, uMgungundlovu district municipality, and traditional leadership and community members for all their support throughout the study. We also thank the study staff for the field work and the study participants, who through their participation provide valuable knowledge and insights on the HIV epidemic in the study area

\section{References}

1 Abdool Karim SS, Abdool Karim Q. Antiretroviral prophylaxis: a defining moment in HIV control. Lancet 2011; 378: 23-25.

2 Cohen MS, Chen YQ, McCauley M, et al. Prevention of HIV-1 infection with early antiretroviral therapy. N Engl J Med 2011; 365: 493-505.

3 Rodger A, Cambiano V, Bruun T, et al. HIV transmission risk through condomless sex if HIV+ partner on suppressive ART: PARTNER study. 21st Conference on Retroviruses and Opportunistic Infections; March 3-6, 2014; Boston, USA Abstract 153LB.

4 Quinn T, Wawer M, Sewankambo N, et al. Viral load and heterosexual transmission of human immunodeficiency virus type 1. Rakai Project Study Group. N Engl J Med 2000; 342: 921-29.

5 Tanser F, Bärnighausen T, Grapsa E, Zaidi J, Newell M-L High coverage of ART associated with decline in risk of HIV acquisition in rural KwaZulu-Natal, South Africa. Science 2013 339: 966-71.

6 Joint United Nations Programme on HIV/AIDS (UNAIDS) 90-90-90 an ambitious treatment target to help end the AIDS epidemic. October, 2014. http://www.unaids.org/sites/default/files/ media_asset/90-90-90_en.pdf (accessed June 28, 2017). 
7 Gardner EM, Young B. The HIV care cascade through time. Lancet Infect Dis 2014; 14: 5-6.

8 Kilmarx PH, Mutasa-Apollo T. Patching a leaky pipe: the cascade of HIV care. Curr Opin HIV AIDS 2013; 8: 59-64.

9 National Department of Health Republic of South Africa. The 2013 National Antenatal Sentinel HIV Prevalence Survey South Africa 2013. 2015. https://www.health-e.org.za/wp-content/ uploads/2016/03/Dept-Health-HIV-High-Res-7102015.pdf (accessed June 28, 2017).

10 Johnson LF. Access to antiretroviral treatment in South Africa, 2004-2011. South Afr J HIV Med 2012; 13: 22-27.

11 Department of Health Republic of South Africa. The South African antiretroviral treatment guidelines 2013 version 14. Pretoria: Department of Health, Republic of South Africa, 2013.

12 Department of Health Republic of South Africa. National consolidated guidelines for the prevention of mother-to-child transmission of HIV (PMTCT) and the management of HIV in children, adolescents and adults. Pretoria: Department of Health, Republic of South Africa, 2014.

13 Kharsany ABM, Cawood C, Khanyile D, et al. Strengthening HIV surveillance in the antiretroviral therapy era: Rationale and design of a longitudinal study to monitor HIV prevalence and incidence in the uMgungundlovu District, KwaZulu-Natal, South Africa BMC Public Health 2015; 15: 1149.

14 Daar ES, Pilcher CD, Hecht FM. Clinical presentation and diagnosis of primary HIV-1 infection. Curr Opin HIV AIDS 2008; 3: $10-15$.

15 Rao JNK, Scott AJ. On chi-squared tests for multiway contingency tables with cell proportions estimated from survey data. Ann Stat 1984; 12: 46-60.

16 Rao JNK, Scott AJ. On simple adjustments to chi-square tests with sample survey data. Ann Stat 1987; 15: 385-97.

17 Heeringa SG, West BT, Berglund PA. Applied survey data analysis. Boca Raton, FL: CRC press, 2010.

18 Gaolathe T, Wirth KE, Holme MP, et al. Botswana's progress toward achieving the 2020 UNAIDS 90-90-90 antiretroviral therapy and virological suppression goals: a population-based survey. Lancet HIV 2016; 3: e221-30.

19 Maman D, Chilima B, Masiku C, et al. Closer to 90-90-90. The cascade of care after 10 years of ART scale-up in rural Malawi: a population study. J Int AIDS Soc 2016; 19: 20673.

20 Maman D, Zeh C, Mukui I, et al. Cascade of HIV care and population viral suppression in a high-burden region of Kenya. AIDS 2015; 29: 1557-65.

21 Justman J, Ellman T, Donnell D, Duong Y, Reed J, Bicego G. Population HIV viral load in Swaziland: assessing ART program effectiveness and transmission potential. 20th Conference on Retroviruses and Opportunistic Infections; March 3-6, 2013; Atlanta, GA, USA. Abstract 96.
22 Kranzer K, Lawn SD, Johnson LF, Bekker L-G, Wood R. Community viral load and CD4 count distribution among people living with HIV in a South African Township: implications for treatment as prevention. J Acquir Immune Defic Syndr 2013; 63: 498-505.

23 Shisana O, Rehle T, Simbayi LC, et al. South African National HIV Prevalence, Incidence and Behaviour Survey, 2012. Cape Town: HSRC Press, 2014.

24 Hensen B, Lewis JJ, Schaap A, et al. Factors associated with HIV-testing and acceptance of an offer of home-based testing by men in rural Zambia. AIDS Behav 2015; 19: 492-504.

25 Takuva S, Brown A, Macleod W, Pillay Y, Delpech V, Puren AJ. Disparities in engagement within HIV care in South Africa. Conference on Retroviruses and Opportunistic Infections; Feb 23-26, 2015; Seattle, WA, USA. Abstract 154.

26 Fidler SJ, Floyd S, Yang B, et al. Towards the second UNAIDS target: population-level ART coverage in HPTN 071 (PopART) 23rd Conference on Retroviruses and Opportunistic Infections Feb 22-25, 2016; Boston, MA, USA. Abstract 114

27 Levi J, Raymond A, Pozniak A, et al. Can the UNAIDS 90-90-90 target be achieved? Analysis of 12 national level HIV treatment cascades. 8th IAS Conference on HIV Pathogenesis, Treatment and Prevention; July 19-22, 2015; Vancouver, BC, Canada. Abstr MOAD0102.

28 Lippman SA, Shade SB, El Ayadi AM, et al. Attrition and opportunities along the HIV care continuum: findings from a population-based sample, North West Province, South Africa. J Acquir Immune Defic Synr 2016; 73: 91-99.

29 South African National AIDS council. Enhanced progress report of the South African national strategic plan on HIV, STIS and TB (2012-2016). March, 2016. http://sanac.org.za/wp-content/ uploads/2016/07/EPR-SANAC-NEW-DOC-latest-update3.pdf (accessed June 28, 2017).

30 Van Rooyen H, McGrath N, Chirowodza A, et al. Mobile VCT: reaching men and young people in urban and rural South African pilot studies (NIMH Project Accept, HPTN 043). AIDS Behav 2013; 17: 2946-53.

31 Shanaube K, Chaila JM, Floyd S, et al. Uptake of HIV testing in the HPTN 071 (PopART) Trial in Zambia. 23rd Conference on Retroviruses and Opportunistic Infections; Feb 22-25, 2016; Boston, MA, USA. Abstract 981. 